\title{
The influence of L-phenylalanine, methyl jasmonate and sucrose concentration on the accumulation of phenolic acids in Exacum affine Balf. f. ex Regel shoot culture*
}

\author{
Ewa Skrzypczak-Pietraszek ${ }^{\bowtie}$, Joanna Słota ${ }^{1}$ and Jacek Pietraszek² \\ 'Chair and Department of Pharmaceutical Botany, Collegium Medicum, Jagiellonian University, Kraków, Poland; 2Institute of Applied Informatics, \\ Cracow University of Technology, Kraków, Poland
}

Phenolic acids are an important group of plant secondary metabolites with various, valuable therapeutic properties. Apart from plants growing in the open air, tissue cultures can be an alternative source of the secondary metabolites. The yield of their accumulation in in vitro cultures can be increased by different methods, including culture medium supplementation with precursors, elicitors and changing the standard amounts of the medium components. The purpose of this study was to investigate the influence of the precursor (L-phenylalanine), the elicitor (methyl jasmonate) and a higher sucrose concentration on the phenolic acids accumulation in the agitated shoot cultures of Exacum affine Balf. f. ex Regel (Gentianaceae). Qualitative and quantitative analyses of the phenolic acids in methanolic extracts from the biomass were conducted by applying the HPLC method. Fourteen phenolic acids and cinnamic acid were found in all samples. The total content of free phenolic acids increased from approximately $0.242 \%$ to $0.635 \%$ ( 2.6 -fold) and the total content of the whole phenolic acids (free and bound) - from $0.712 \%$ to $1.160 \%$ (1.6-fold). The studies show that the best variant for the accumulation of most of the identified phenolic acids contained $6 \%$ of sucrose (double the standard amount), L-phenylalanine $1.6 \mathrm{~g} \mathrm{~L}^{-1}$ of medium and methyl jasmonate $100 \mu \mathrm{M}$. The analysis of the results in the experiment presented here showed that it is possible to increase the accumulation of the phenolic acids in Exacum affine shoot cultures by adding the precursor (L-phenylalanine), the elicitor (methyl jasmonate) and by increasing the sucrose concentration.

Key words: Exacum affine Balf. f. ex Regel, Persian violet, elicitation, precursor feeding, L-phenylalanine, methyl jasmonate, sucrose concentration

Received: 25 November, 2013; revised: 21 February, 2014; accepted: 21 February, 2014; available on-line: 18 March, 2014

\section{INTRODUCTION}

Exacum affine Balf. f. ex Regel belongs to the Gentianaceae. There are 65 Exacum species spreading from westernmost Africa to Madagascar and Socotra, and from India to New Guinea. E. affine is endemic to Socotra (Yuan et al., 2005). This species is known in Europe and North America as a beautiful potted plant (names: Persian gentian; Persian, Arabian or German violet). Of the all species of Exacum, only E. affine has been suc- cessfully domesticated and so far gained economic importance as an ornamental crop (Riseman, 2006). Apart from the floriculture use, we might notice some interest in E. affine's phytochemical constituents and its therapeutic activity. E. affine was mentioned by Mothana and coresearchers (Mothana \& Lindequist, 2005; Mothana et al., 2006; Mothana et al., 2007) in their ethnopharmacobotanical studies published in recent years. E. affine showed strong antiviral activity (Mothana et al., 2006). This species has been investigated as one of the 25 medicinal plants which are used in Yemeni traditional medicine to treat infectious illnesses (e.g. skin and respiratory tract infections). The results demonstrate that E. affine shows the significant antiviral activity against herpes simplex virus (HSV-1 strain KOS) and human influenza virus A/ WSN/33 (H1N1) London. These results confirm the traditional use of the plant against viral infections. Mothana et al. (2006) also suggest that phenolic acids may be one of the chemical groups responsible for such activity.

Hegnauer (1966) mentioned p-courmaric acid as a constituent of E. affine. Skrzypczak-Pietraszek (unpublished data) analysed E. affine pot plants and found fourteen phenolic acids (caffeic, chlorogenic, ferulic, gallic, gentisic, o-coumaric, $p$-coumaric, $p$-hydroxybenzoic, protocatechuic, rosmarinic, salicylic, sinapic, syringic, vanillic). Most of the compounds were only in the bound form and were detected after acid hydrolysis. Protocatechuic acid was the main phenolic acid of E. affine herb. Only few information relate to the phenolic acid content in the other species of the genus Exacum. Daniel and Sabnis (1978) made the qualitative analysis of E. bicolor and E. pedunculatum. They found four phenolic acids in $E$. bicolor ( $p$-coumaric, $p$-hydroxybenzoic, protocatechuic, vanillic) and six compounds in E. pedunculatum (vanillic, syringic, $p$-hydroxybenzoic, protocatechuic, $p$-coumaric, ferulic). Jeeshna and Paulsamy (2011) qualtitatively determined the chlorogenic acid content in E. bicolor.

Phenolic acids are the significant constituents of secondary metabolites widespread in the plant kingdom, showing - apart from the antiviral action - a broad spectrum of therapeutic activities, such as antiseptic, antioxidant, antiphlogistic, cholagogic, immunostimulating,

e-mail: ewa.skrzypczak-pietraszek@uj.edu.pl

* Preliminary report on the same subject presented at scientific meeting at 5th Central European Congress of Life Sciences EUROBIOTECH 2013, Kraków, Poland

Abbreviations: BAP, 6-benzylaminopurine; DW, dry weigth; $\mathrm{GA}_{3}$, gibberellic acid; MeJA, methyl jasmonate; MS, Murashige and Skoog (1962) medium; NAA, a-naphthaleneacetic acid; Phe, L-phenylalanine; Suc, sucrose 
spasmolytic and others (Khadem \& Marles, 2010). Phenolic acids biosynthesis is based on a shikimate-phenylpropanoid pathway. Apart from their potential pharmacological activities, these compounds play an important role as active defence factors in the plants' resistance to diseases and other biotic and also abiotic stresses. The phenylpropanoid pathway is often induced by stress and has a specific function in plant protection (Arora, 2010).

The biotechnological production of the secondary metabolites in plant cell and organ cultures is an attractive alternative to the extraction of the whole plant material. Numerous cell and tissue cultures have been derived from many medicinal plants but they do not often produce sufficient amounts of the required compounds. For this reason, many biotechnological strategies have been prepared and experimented for the enhanced production of secondary metabolites from medicinal plants. Some of them include the screening of high yielding cultures, media modifications, the precursor feeding, elicitation and others (Namdeo, 2007; Arora, 2010).

Sucrose is one of the standard component of the culture medium and it is an important carbon and energy source for the plant cells. In addition, the sucrose content influences the production of secondary metabolites of the phenylpropanoid pathway (Arora, 2010).

Phenylalanine (Phe) is an amino acid, the precursor of the phenylpropanoide pathway leading to the formation of phenolic acids, flavonoids and other phenolic compounds. Phenylalanine has been successfully used to increase the metabolite production in vitro in many different plant cultures (Arora, 2010).

The methyl jasmonate (MeJA) is one of the abiotic elicitors. It activates phenylalanine ammonia lyase (the enzyme that catalyzes the first step in the shikimic acid pathway - the deamination of Phe) and thus induces the secondary metabolites production (Namdeo, 2007).

According to our knowledge, there was no literature information on the elicitation and the precursor feeding experiments in the in vitro cultures of the plants from the genus Exacum. Some publications concerned the other taxons from the family Gentianaceae. The influence of the methyl jasmonate, L-phenylalanine (Phe) or the sucrose concentration on the accumulation of iridoids or xanthones in Swertia chirata (Keil et al. 2000; Kumar et al. 2013) Centaurium sp. (Abd El-Mawla et al. 2001; Beerhues and Berger 1995) in vitro cultures.

The purpose of this study was to investigate the influence of the precursor (Phe), the elicitor (MeJA) and the different sucrose concentrations on the phenolic acids accumulation in the agitated shoot cultures of Exacum affine Balf. f. ex Regel (Gentianaceae).

\section{MATERIALS AND METHODS}

Plant material. E. affine potted plants were bought in 2009 from the commercial specialized horticultural company (F.P.H. "Ogród Lęg" s.c. B.J.T. Gawęda, Kraków, Poland). The plants were at an early flowering phase. Plants identity was verified by Dr. Skrzypczak-Pietraszek, referring to available literature. A voucher specimen was deposited in the Department of Pharmaceutical Botany (Jagiellonian University, Collegium Medicum, Kraków, Poland) with the number 2009-EA01. Plants were used to establish in vitro cultures.

In vitro cultures. Shoot cultures induction and culture conditions. Shoot cultures were established in 2009. Stems from potted plants (see: Plant material) were used as initial explants. Stems were surface- sterilized with $70 \%(\mathrm{v} / \mathrm{v})$ ethanol for $0.5 \mathrm{~min}$, followed by $0.2 \% \mathrm{HgCl}_{2}$ treatment for $20 \mathrm{~min}$. Then, they were rinsed three times with sterile distilled water, cut into $1.0-1.5 \mathrm{~cm}$ pieces and transferred onto previously selected (Skrzypczak-Pietraszek et al., 2005) the MS (Murashige \& Skoog, 1962) medium, supplemented with $1.0 \mathrm{mg} \mathrm{L}^{-1}$ BAP (6-benzylaminopurine), $0.5 \mathrm{mg} \mathrm{L}^{-1} \mathrm{NAA}$ $\left(\alpha\right.$-naphthaleneacetic acid), $0.25 \mathrm{mg} \mathrm{L}^{-1} \mathrm{GA}_{3}$ (gibberellic acid). The MS medium contained $3 \%$ sucrose (w/v) and was solidified with $0.8 \%$ agar (w/v). After 1-2 weeks, green callus appeared on the explants and differentiated into the shoot culture during 1-2 subcultures. Shoot cultures were grown in $100 \mathrm{~mL}$ Erlenmeyer flasks in a culture room at $25 \pm 2^{\circ} \mathrm{C}$ under constant artificial light (16 $\left.\mu \mathrm{mol} \cdot \mathrm{m}^{-2} \cdot \mathrm{s}^{-1}\right)$ and were transferred onto the same fresh medium every 4-5 weeks.

Agitated shoot culture. In order to perform the experiment, $21^{\text {th }}$ subculture of E. affine shoot cultures was used to establish an agitated shoot culture in $500 \mathrm{~mL}$ Erlenmeyer flasks ( $4 \mathrm{~g}$ of fresh shoots/flask) on a rotary shaker at $140 \mathrm{rpm}$ and in the same other culture conditions. The MS medium was supplemented with the same growth regulators. The medium was liquid (without agar) and contained a standard content of sucrose (Suc) $3 \%(\mathrm{w} / \mathrm{v})$ (variant $\mathrm{A}^{\prime}$ - reference) or double amount of Suc $-6 \%(\mathrm{w} / \mathrm{v})$ (A-F variants). Agitated cultures were maintained for 14 days and then they were treated with Phe and MeJA.

Elicitation and precursor feeding experiment. The stock solutions of Phe (Merck) and MeJA (Sigma-Aldrich) were prepared: Phe was dissolved in water, MeJA in $96 \%(\mathrm{v} / \mathrm{v})$ ethanol and both were filter-sterilised (Milligore Millex-GP, $0.22 \mu \mathrm{m})$. The precursor and elicitor were added to the agitated shoot culture on the $14^{\text {th }}$ day. Phe achieved a final concentration of $1.6 \mathrm{~g} \mathrm{~L}^{-1}(\approx 10$ $\mathrm{mM}$ ) and MeJA: $100 \mu \mathrm{M}$ or $800 \mu \mathrm{M}$. Phe and MeJA were added to the cultures independently or in a combination, according to the following scheme:

$$
\begin{aligned}
& \text { - variant } \mathrm{B} \text { - MeJA } 100 \mu \mathrm{M} \\
& \text { - variant } \mathrm{C} \text { - MeJA } 800 \mu \mathrm{M} \\
& \text { - variant D - Phe } 1.6 \mathrm{~g} \mathrm{~L}-1 \\
& \text { - variant } \mathrm{E} \text { - Phe } 1.6 \mathrm{~g} \mathrm{~L}-1 \text {; MeJA } 100 \mu \mathrm{M} \\
& \text { - variant } \mathrm{F} \text { - Phe } 1.6 \mathrm{~g} \mathrm{~L}^{-1} \text {; MeJA } 800 \mu \mathrm{M}
\end{aligned}
$$

Variants A' and A were treated as controls. Variants without Phe or/and MeJA were supplemented with sterile water or ethanol, respectively. Ethanol achieved a final concentration of $0.1 \%(\mathrm{v} / \mathrm{v})$. After elicitation and precursor feeding, all the cultures were placed again on the rotary shaker. The biomass was harvested after 1, 3 and 7 days, frozen and lyophilised. The dried material was used for the phytochemical analysis.

\section{Phytochemical analysis}

General experimental procedures. Methanol and acetic acid (both of HPLC grade) were purchased from Merck (Darmstadt, Germany). The phenolic acid standards were from Fluka and Sigma. The water was purified by re-distillation followed by filtration through Millipore $0.22-\mu \mathrm{m}$ filters. The standard solutions or the studied samples were filtered through $0.22-\mu \mathrm{m}$ syringe filters (Millipore, Millex-GP) before being injected to HPLC. The qualitative and quantitative HPLC analyses of phenolic acids were conducted using VWR Hitachi apparatus (autosampler L 2200, pump L 2130, column oven L 2350, diode array detector (DAD) L 2455) at UV range 200-400 nm and Purospher ${ }^{\circledR}$ RP-18e column $(250 \times 4$ $\mathrm{mm}, 5 \mu \mathrm{m})$ thermostated at $25^{\circ} \mathrm{C}$. A mobile phase was composed of methanol $-0.5 \%$ acetic acid $(1: 4, \mathrm{v} / \mathrm{v})$ 
as solvent $\mathrm{A}$ and methanol (solvent B). The following gradient elution scheme was used (A:B ratio): 100\%:0\% $\mathrm{t}=0-20 \mathrm{~min} ; 80 \%: 20 \% \mathrm{t}=35 \mathrm{~min} ; 70 \%: 30 \% \mathrm{t}=45 \mathrm{~min}$; 60\%:40\% $\mathrm{t}=55 \mathrm{~min} ; 50 \%: 50 \% \quad \mathrm{t}=60 \mathrm{~min} ; 25 \%: 75 \%$ $\mathrm{t}=65 \mathrm{~min} ; 0 \%: 100 \% \mathrm{t}=70-75 \mathrm{~min} ; 100 \%: 0 \% \mathrm{t}=80-90$ $\min$.

Extraction. The extraction process was carried out as follows: $1 \mathrm{~g}$ of each dried powdered (in a mortar) plant material was extracted twice with $50 \mathrm{~mL}$ of boiling methanol $\left(\right.$ at $67.4^{\circ} \mathrm{C}$ ) for two hours under reflux condenser. The combined extracts $(100 \mathrm{~mL})$ were concentrated to dryness, using a rotary vacuum evaporator (Büchi) at $40^{\circ} \mathrm{C}$. The residues were dissolved in $5 \mathrm{~mL}$ of methanol. The extract samples contained free phenolic acids.

Hydrolysis. The hydrolysis process was carried out as follows: $1 \mathrm{~g}$ of each dried powdered (in a mortar) plant material was hydrolysed with $50 \mathrm{~mL}$ of $2 \mathrm{M}$ hydrochloric acid for two hours at $100^{\circ} \mathrm{C}$. The hydrolysates were extracted with ethyl acetate (5 times with $40 \mathrm{~mL}$ of ethyl acetate). The acetate extracts were combined $(200 \mathrm{~mL})$ and concentrated to dryness in a rotary vacuum evaporator (Büchi) at $40^{\circ} \mathrm{C}$. The hydrolysed samples contained all phenolic acids present in the plant material: free and earlier bound with other plant metabolites. The residues were dissolved in $5 \mathrm{~mL}$ of methanol.

HPLC-DAD analysis. A HPLC method was applied according to the procedure developed by Ellnain-Wojtaszek \& Zgorka (1999). The comparison of UV spectra and retention times with standard compounds enabled the identification of phenolic acids present in the analysed samples. The qualitative analyses were supplemented with an internal standard method. The phenolic acids identified by the analyses were quantified against their external standards (the calibration curve method).

Statistical methods. The statistical analysis usually requires data pre-processing, e.g. an outliers detection or a reduction of dimensionality through the non-parametric data-driven procedures: the cluster analysis (CA) (Everitt et al., 2011) or the principle component analysis (PCA) (Jolliffe, 2010). In the research described here, the data pre-processing was not necessary, contrary to the author's previous experience (Skrzypczak-Pietraszek \& Pietraszek, 2012).

The multiple comparison of means was conducted by applying a classic oneway analysis of variance (ANOVA) (Fisher, 1935; Kempthorne \& Hinkelmann, 2007). The equality of variances across samples, usually called a homoscedascity or a homogeneity of variances, is one of the basic assumptions in ANOVA. The typical test of such equality is Bartlett's test (Bartlett, 1937) but it requires the normality of general population. Such an assumption could not be reliably tested by any test of normality e.g. Shapiro-Wilk (Shapiro \& Wilk, 1965) or Anderson-Darling (Anderson \& Darling, 1954) due to a small size of the samples obtained. This specific situation led to the Levene's test (Levene, 1960), which does not require the normality of general population.

When the test response was negative (the critical p-Value less than the assumed significance level alpha), the Box-Cox transformation (Box \& Cox, 1964) was used to achieve a better balance of variances. When the Levene's test response for transformed data was non-negative, ANOVA was evaluated. If the Levene's test was still negative, the non-parametric variant of ANOVA (Kruskal \& Wallis, 1952) was evaluated for original data. The power of non-parametric ANOVA is smaller than that of classic parametric ANOVA but it does not require any equality of variances across samples. The results of the statistical analysis were illustrated by a box-plot.

\section{RESULTS AND DISCUSSION}

In the present experiment, the agitated shoot culture of Exacum affine was maintained in the MS medium supplemented with BAP $1 \mathrm{mg} \mathrm{L}^{-1}$, NAA $0.5 \mathrm{mg} \mathrm{L}^{-1}$ and $\mathrm{GA}_{3} 0.25 \mathrm{mg} \mathrm{L}^{-1}$. The medium A' contained 3\% sucrose, which is the standard content, while the remaining media $\mathrm{A}, \mathrm{B}, \mathrm{C}, \mathrm{D}, \mathrm{E}$ and $\mathrm{F}$ were enriched with $6 \%$ sucrose. After 14-day culture in the media $\mathrm{BF}$, the precursor (Phe) and elicitor (MeJA) were added, according to the scheme presented. The media A and A' were treated as controls. The plant material was collected from cultures 1,3 and 7 days after the precursor and/or the elicitor had been added.

\section{The effect of medium composition on the biomass growth}

The comparison of the dry weights demonstrated that the biomass growth on the media A and A' was the lowest after the first day and the highest after 7 days, as expected (Fig. 1).

Adding the precursor (Phe) to the variant $\mathrm{D}$ of the medium initially stimulated the biomass growth in a culture, however a slowdown was observed after 7 days. A similar relationship was noted for the variant B (supplemented with MeJA $-100 \mu \mathrm{M}$ ) and the variant $\mathrm{E}$ (combined treatment with Phe and MeJA - $100 \mu \mathrm{M}$ ). Methyl jasmonate at $800 \mu \mathrm{M}$ (variants C and F) markedly suppressed the culture growth. The slight browning of shoots was observed especially after 7 days, which can indicate even a tissue damage. Our observations, indicating the inhibitory effect of MeJA on the biomass growth, are similar to others known from the literature (Namdeo, 2007; Kikowska et al., 2012).

\section{Qualitative analysis of phenolic acids}

All analyzed extracts were found to contain 14 phenolic acids, including 5 cinnamic acid derivatives and 7 benzoic acid derivatives. Cinnamic acid was identified, as well. The results of the present experiment demonstrated that Phe was present in all biomass extracts and

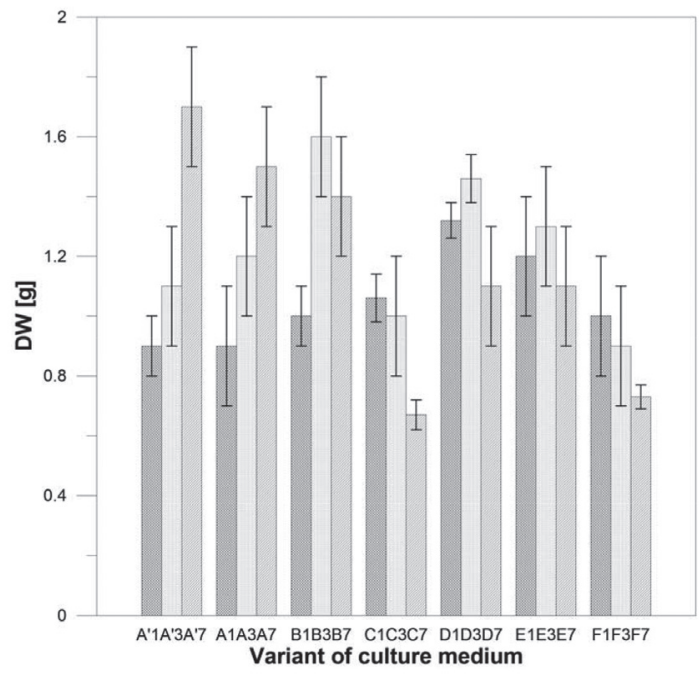

Figure 1. Dry weigth of the biomass collected from E. affine agitated shoot cultures.

Data are expressed as mean \pm standard error $(n=3)$. Variants of media: A' (Suc - 3\%); A (Suc - 6\%); B (MeJA - $100 \mu \mathrm{M}$; Suc $6 \%) ; C$ (MeJA - $800 \mu \mathrm{M}$; Suc $-6 \%) ; D\left(P h e-1.6 \mathrm{~g} \mathrm{~L}^{-1}\right.$; Suc $6 \%) ; \mathrm{E}$ (Phe - $1.6 \mathrm{~g} \mathrm{~L}^{-1}$; MeJA - $100 \mu \mathrm{M}$; Suc - 6\%); F (Phe $1.6 \mathrm{~g} \mathrm{~L}^{-1} ;$ MeJA $-800 \mu \mathrm{M}$; Suc $-6 \%$ ). 

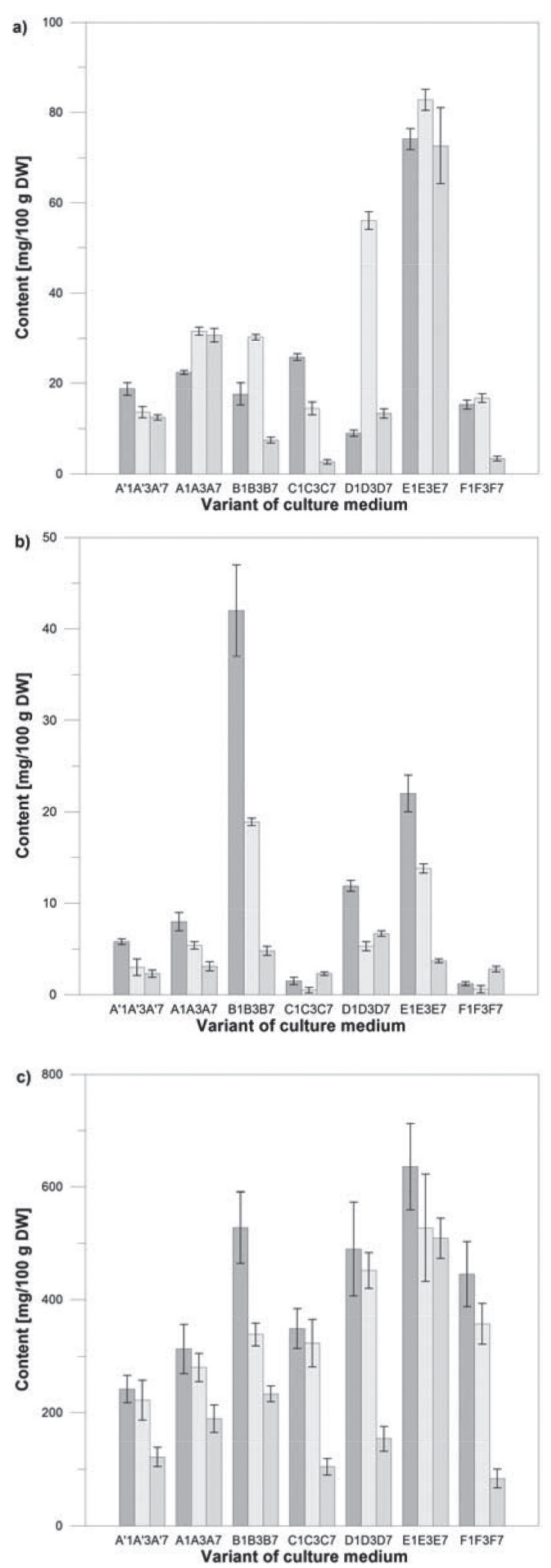

Figure 2. Content of the free phenolic acids in the biomass collected from E. affine shoot cultures: (a) chlorogenic acid, (b) caffeic acid, (c) total phenolic acids content (the sum of all identified compounds).

Data are expressed as mean \pm standard error $(n=3)$. Variants of media: A' (Suc - 3\%); A (Suc - 6\%); B (MeJA - $100 \mu \mathrm{M}$; Suc $6 \%) ; C$ (MeJA - $800 \mu \mathrm{M}$; Suc - 6\%); D (Phe - $1.6 \mathrm{~g} \mathrm{~L}^{-1}$; Suc $6 \%) ; \mathrm{E}$ (Phe - $1.6 \mathrm{~g} \mathrm{~L}^{-1}$; MeJA - $100 \mu \mathrm{M}$; Suc - 6\%); F' (Phe $1.6 \mathrm{~g} \mathrm{~L}^{-1} ; \mathrm{MeJA}-800 \mu \mathrm{M}$; Suc - 6\%). post culture media. Notably, none or only trace amounts of phenolic acids were detected in the media. The identity of the compounds was confirmed by comparing the retention times and UV spectra of the test substances with the respective parameters of the reference standards. The internal standard method was applied as well.

\section{Quantitative analysis of phenolic acids}

The comparison of the total contents of phenolic acids in the study plant material (Fig. 2 and Fig. 3) demonstrated a wide variation, especially among free phenolic acids. Adding Phe (variant D) or MeJA (variant B) stimulated the accumulation of the studied compounds. The treatment combined with both compounds (variant E) was very beneficial. It was important to choose correctly the MeJA concentration because this substance can be harmful for the biomass cultured in vitro. It was observed that a higher elicitor concentration of $800 \mu \mathrm{M}$ caused such a disadvantageous effect. The concentration of Phe (1.6 $\mathrm{g} \mathrm{L}^{-1}$ medium) was selected on the basis of earlier studies (Skrzypczak-Pietraszek unpublished data). MeJA was added to $E$. affine shoot cultures for the first time, so its concentrations $(100 \mu \mathrm{M}$ and $800 \mu \mathrm{M})$ were chosen on the basis of literature data, indicating that suspension cultures were more sensitive to the toxic effect of MeJA than organ (e.g. shoot) cultures (Namdeo, 2007; Kikowska et al., 2012). It should also be remembered that too low elicitor concentration will not produce a stimulating effect on the accumulation of secondary metabolites.

The experiment demonstrated that the variant $\mathrm{E}$ containing $6 \%$ sucrose (twice higher a concentration than a standard value), the precursor Phe (1.6 $\mathrm{g} \mathrm{L}^{-1}$ medium) and the elicitor MeJA $(100 \mu \mathrm{M})$. was the most beneficial of the tested variants. This medium variant was especially stimulating for the accumulation of some phenolic acids (e.g. chlorogenic acid (Fig. 2a, Fig. 3a) and 4-hydroxybenzoic acid), both in the bound and free form.

Other phenolic acids (e.g. syringic acid and vanillic acid) showed a wide variability of the free form content in response to stimulation by different medium variants supplemented with the precursor and elicitor, while the total accumulation of the compound (free + bound in extracts after hydrolysis) differed much less. It can be assumed that some variants (e.g. variant E) are particularly beneficial to the accumulation of phenolic acids in the free form, while the total biosynthetic capability is not increased so much. In other medium variants, derivatives of phenolic acids were the dominating form.

The largest quantities of most of the tested phenolic acids were accumulated in shoots cultured on variant $\mathrm{E}$, but there were some exemptions, e.g. variant B1 was the most beneficial to caffeic acid (Fig. 2b, Fig. 3b) and $o$-coumaric acid accumulation (both acids in the free form).

The greatest increase in the free form accumulation $v s$. control was observed for:

- synapic acid - 9.7-fold,

- o-coumaric acid - 9.4-fold,

- caffeic acid - 7.2-fold (Fig. 2b),

- p-coumaric acid - 7.2-fold,

- chlorogenic acid - 6.0-fold (Fig. 2a),

- vanillic acid - 5.8-fold,

whereas the greatest increase of the total content (free plus bound) was noted for :

- synapic acid - 7.7-fold,

- chlorogenic acid - 7.3-fold (Fig. 3a),

- 4-hydroxybenzoic acid - 3.6-fold. 

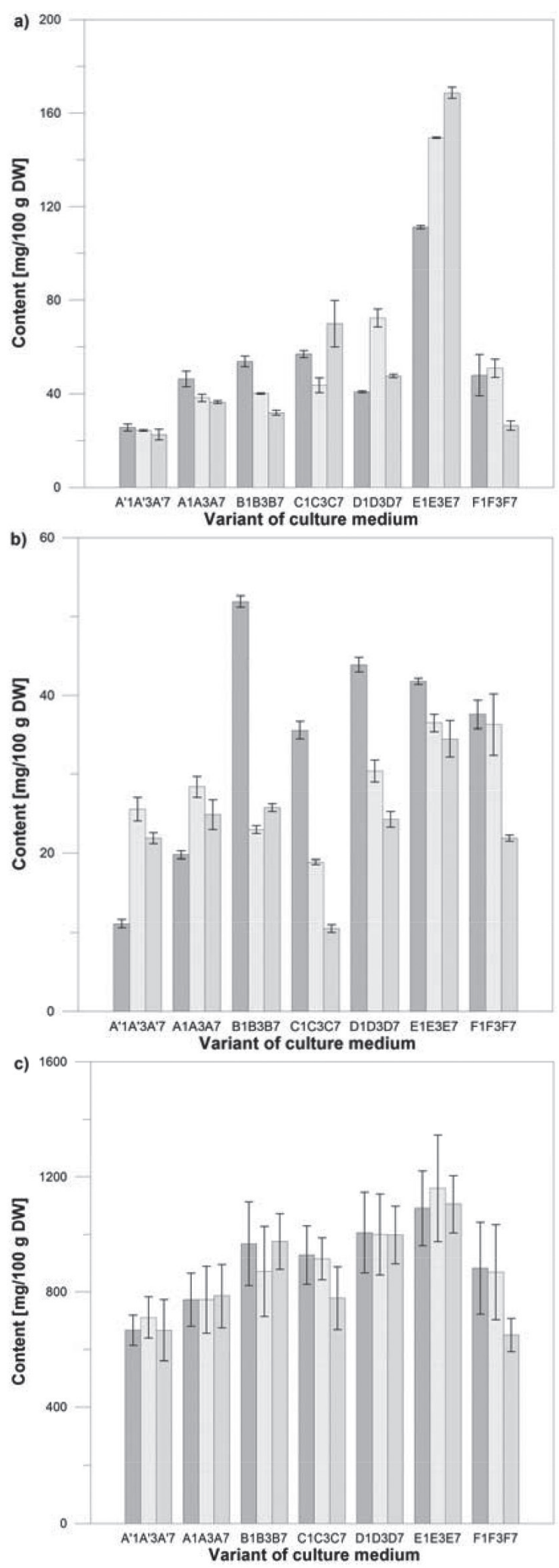

Figure 3. Content of phenolic acids (free and bound - samples after hydrolysis) in the biomass collected from $E$. affine agitated shoot cultures: (a) chlorogenic acid, (b) caffeic acid, (c) total phenolic acids content (the sum of all identified compounds). Data are expressed as mean \pm standard error $(n=3)$. Variants of media: A' (Suc - 3\%); A (Suc - 6\%); B (MeJA - $100 \mu \mathrm{M}$; Suc $6 \%)$; C (MeJA - $800 \mu \mathrm{M}$; Suc - 6\%); D (Phe - $1.6 \mathrm{~g} \mathrm{~L}^{-1}$; Suc 6\%); E (Phe - $1.6 \mathrm{~g} \mathrm{~L}^{-1} ;$ MeJA - $100 \mu \mathrm{M}$; Suc - 6\%); F' (Phe $1.6 \mathrm{~g} \mathrm{~L}^{-1}$; MeJA $-800 \mu \mathrm{M}$; Suc - 6\%).
The total content of free phenolic acids increased 2.6fold and the total content of the whole phenolic acids (free and bound) -1.6-fold (Fig. 2c, Fig. 3c).

Not only the addition of the precursor or elicitor but also the time of shoot harvesting influenced phenolic acid accumulation. The highest content was often noted on the first day after medium supplementation, which was usually followed by a decrease in metabolite accumulation on the seventh day. Some plants, e.g. Oryza sativa, contain two isoforms of the enzyme. One of these isoforms - phenylalanine ammonia lyase - is involved in the elicitor-induced response (Giberti et al., 2012) and it exhibits its activity twice: 6 hours and then 48-72 hours after elicitation. Perhaps, it is similar for E. affine. The highest phenolic acid concentrations are observed one or three days after elicitation and then their contents decrease because they can be converted into different phenolic compounds by other enzymes involved in the phenylpropanoid pathway.

Comparing the medium variants: A' $3 \%(\mathrm{w} / \mathrm{v})$ of Suc) and A (6\% (w/v) of Suc), it can be noticed that a higher concentration of Suc increases the phenolic acids accumulation. Similar results were mentioned by other authors, e.g. (Namdeo, 2007; Kikowska et al., 2012).

On the other hand, Keil et al. (2000) found that the addition of Suc at $6 \%$ and $9 \%(\mathrm{w} / \mathrm{v})$ resulted in the better growth rates of root cultures of Swertia chirata (Gentianaceae), but had no influence on the relative amarogentin content compared to $3 \%(\mathrm{w} / \mathrm{v})$ Suc. Numerous elicitors, precursors of amarogentin biosynthesis and plant growth regulators were tested, but only chitosan increased the amarogentin accumulation. Shoot cultures of Swertia chirata (Kumar et al., 2013) treated with 50 and $75 \mathrm{uM}$ MeJA showed a significant inhibition of the biomass production and iridoids (swertiamarin, mangiferin, amarogentin) accumulation compared to the control. The lowest MeJA concentration, $25 \mu \mathrm{M}$ - influenced the significant increase of the analysed compounds. The influence of elicitors and precursors on the accumulation of the other important Gentianaceae secondary metabolites - xanthones - in cell cultures of Centaurium sp. was investigated (Beerhues \& Berger, 1995; El-Mawla et al., 2001). Different elicitors induced the formation of two different xanthones: 1-hydroxy-3,5,6,7-tetramethoxyxanthone (MeJA) or 1,5-dihydroxy-3-methoxyxanthone (yeast extract) (Beerhues \& Berger, 1995). In the other experiment, three radiolabelled precursors (Phe, benzoic acid, 3-hydroxybenzoic acid) were incorporated into xanthones in Hypericum androsaemum cells, whereas only one of them (3-hydroxybenzoic acid) was incorporated into Centaurium erythraea xanthones. MeJA can increase the accumulation of the compounds from different chemical groups: e.g. iridoids, xanthones (earlier mentioned), diterpenoids (e.g.Grzegorczyk \& Wysokinska, 2009), phenolic acids (e.g. Grzegorczyk \& Wysokinska, 2009; Kikowska et al., 2012), flavonoids (e.g. Thiem \& Krawczyk, 2010), alkaloids (e.g. Jaremicz et al., 2014). It is interesting to observe the MeJA influence on the accumulation of different chemical compounds in one experiment (e.g. Grzegorczyk \& Wysokinska, 2009). The maximum level of diterpenoids in shoot cultures of Salvia officinalis was achieved at 3 days after elicitation with $20 \mu \mathrm{M}$ MeJA, but the highest amount of rosmarinic acid was observed on the 5th day after elicitation (50 or $100 \mathrm{uM} \mathrm{MeJA}$ ).

Some authors suggest the beneficial effect of the simultaneous addition of the elicitor and the precursor e.g. (Krolicka et al., 2008; Qu et al., 2011; Riedel et al., 2012). Our results with MeJA/Phe confirm these observations. On the other hand, sometimes the elicitor/precursor 

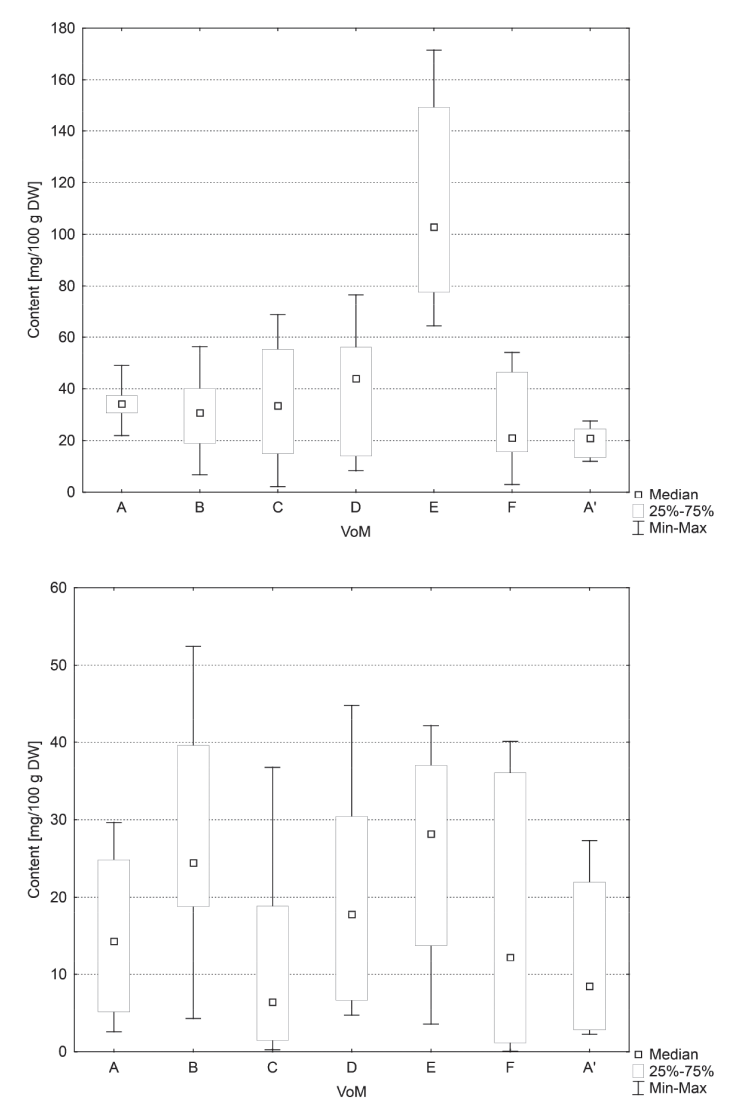

Figure 4. Non-parametric Kruskal-Wallis ANOVA (a - chlorogenic acid, b - caffeic acid)

Data are expressed as mean \pm standard error $(n=3)$. Variants of media: A' (Suc - 3\%); A (Suc - 6\%); B (MeJA - $100 \mu \mathrm{M}$; Suc 6\%); C (MeJA - $800 \mu \mathrm{M}$; Suc - 6\%); D (Phe - $1.6 \mathrm{~g} \mathrm{~L}^{-1}$; Suc 6\%); E (Phe - $1.6 \mathrm{~g} \mathrm{~L}^{-1} ;$ MeJA - $100 \mu \mathrm{M}$; Suc - 6\%); F' (Phe $1.6 \mathrm{~g} \mathrm{~L}^{-1} ;$ MeJA $-800 \mu \mathrm{M}$; Suc $\left.-6 \%\right)$.

combinations do not influence the secondary metabolites accumulation (Cousins et al., 2010).

Apart from elicitors, precursors, osmotic stress (higher concentrations of Suc), there are much more factors which may influence the accumulation of phenolic acids and other secondary metabolites in cell and tissue cultures. These factors include: type of culture medium e.g. MS, $1 / 2$ MS, B5 (Gamborg et al., 1968), 1/2 B5 etc. (e.g. Weremczuk-Jeżyna et al., 2013), different combinations and concentrations of plant growth regulators (e.g. Kumar et al., 2013), the method of the lighting (e.g. Weremczuk-Jeżyna et al., 2013).

\section{Statistical analysis}

The experiment was conducted according to the three-way ANOVA of the main effects scheme. The two responses which were analyzed were the content of caffeic and chlorogenic acids. The classification factors included: the status of the sample (STATUS: BH - before hydrolysis, $\mathrm{AH}$ - after hydrolysis), the variant of the medium (VoM: A', A, B, C, D, E, F) and the day of harvesting (DAY: 1, 3, 7). The whole scheme consisted of 42 groups, each containing 3 numerical measurements (triplicate) taken at the same settings of STATUS, VoM and DAY.

The first analysed variable was the content of the chlorogenic acid. The test of variance homogeneity returned a positive response for DAY ( $p$-Value $=0.44)$ but negative responses for STATUS $(p-$ Value $=0.03)$ and VoM $(p-$ Value $=0.00)$. The Box-Cox transformation returned the value of optimal $\lambda=0.212$, but unfortunately the test of variance homogeneity failed again (STATUS p-Value $=0.03 ;$ VoM p-Value $=0.00 ;$ DAY $\mathrm{p}$-Value $=0.03)$. Due to the violation of the basic ANOVA assumptions, the classical parameter ANOVA could not be processed and the alternative, non-parametric ANOVA proposed by Kruskall and Wallis was involved for each of the grouping factors separately. The results obtained for STATUS are evident (Fig. 4a): STATUS = $\mathrm{BH}$ differs from STATUS $=\mathrm{AH}$ with the significance level $\mathrm{p}$-Value lower than $3 \cdot 10^{\circ}$. The results obtained for $\mathrm{VoM}$ revealed that $\mathrm{VoM}=\mathrm{E}$ differs significantly from other analysed groups. The critical level of significance (p-Value) for multiple comparisons with E medium were lower than 0.01 for all other groups. The pairs involving other groups than E medium were not significantly different. The results obtained for DAY revealed that DAY is not a statistically significant differentiating factor. The impacts of DAY $=1$ and DAY $=3$ are practically indistinguishable: $\mathrm{p}-$ Value $=1.00$ in the multiple comparisons test. The DAY $=7$ is slightly different, however, it is not statistically significant: $\mathrm{p}$-Value with respect to DAY $=1$ is 0.44 and to DAY $=3$ is 0.08 .

The second analysed variable was the content of the caffeic acid. The test of variance homogeneity returned a positive response for STATUS ( $\mathrm{p}-\mathrm{Value}=0.09$ ) but negative responses for DAY $(\mathrm{p}-$ Value $=0.00)$ and $\mathrm{VoM}$ $(\mathrm{p}-$ Value $=0.02)$. The Box-Cox transformation returned the value of optimal $\lambda=0.555$, but unfortunately the test of variance homogeneity failed again (STATUS pValue $=0.19 ;$ VoM $\mathrm{p}$-Value $=0.00 ;$ DAY $\mathrm{p}$-Value $=$ $0.15)$. Due to the violation of the basic ANOVA assumptions, the classical parameter ANOVA could not be processed and the alternative, non-parametric ANOVA proposed by Kruskall and Wallis was involved for each of the grouping factors separately. The results obtained for STATUS are evident (Fig. 4b): STATUS = BH differs from STATUS $=\mathrm{AH}$ with the significance level $\mathrm{p}$ Value lower than $1 \cdot 10^{16}$. The results obtained for VoM revealed that $\operatorname{VoM}=\mathrm{C}$ differs significantly in comparison to $\operatorname{VoM}=\mathrm{B}(\mathrm{p}-$ Value $=0.01)$ and $\operatorname{VoM}=\mathrm{E}(\mathrm{p}-$ Value $=0.02)$, however, other possible comparisons are not significantly different. It should be noted that the comparison between $\mathrm{VoM}=\mathrm{A}^{\prime}$ and $\mathrm{VoM}=\mathrm{B}$ has the value of the critical significance level only slightly greater than the threshold $\mathrm{a}=0.05$. It is 0.07 , which suggests that this pair could be considered as slightly different, for the fact that the threshold is not a strictly sharp decision switch, but rather fuzzy. The results obtained for $\mathrm{DAY}$ revealed that one pair: $\mathrm{DAY}=1$ and $\mathrm{DAY}=7$ significantly differs. The critical significant level $\mathrm{p}$-Value is equal to 0.02 . Other pair comparisons $(1$ vs. 3 and 3 vs. 7) are not significantly different.

\section{CONCLUSIONS}

The analysis of the obtained results showed that the accumulation of phenolic acids in shoot cultures of $E$. affine could be increased by elevating the sucrose concentration in the medium and adding the precursor Phe and the elicitor MeJA. The present experiment indicated that the medium variant $\mathrm{E}$, containing $6 \%$ sucrose (twice as much as the standard value), supplemented with the precursor Phe (1.6 $\mathrm{g} \mathrm{L}^{-1}$ medium) and elicitor MeJA (100 $\mu \mathrm{M})$ was the most efficient to increase phenolic acid accumulation. 


\section{REFERENCES}

Abd El-Mawla AMA, Schmidt W, Beerhues L (2001) Cinnamic acid is a precursor of benzoic acids in cell cultures of Hypericum androsaemum L. but not in cell cultures of Centaurium erythraea RAFN. Planta 212: 288-293.

Anderson TW, Darling DA (1954) A Test of Goodness of Fit. J Am Stat Assoc 49: 765-769.

Arora R (2010) Medicinal Plant Biotechnology. CABI, Wallingford, Oxfordshire, UK.

Bartlett MS (1937) Properties of sufficiency and statistical tests. J R Stat Soc 160: 268-282.

Beerhues L, Berger U (1995) Differential accumulation of xanthones in methyl-jasmonate and yeast-extract-treated cell cultures of Centaurium erythraea and Centaurium littorale. Planta 197: 608-612.

Box GEP, Cox DR (1964) An Analysis of Transformations. J Roy Stat Soc B 26: 211-252.

Cousins MM, Adelberg J, Chen F, Rieck J (2010) Secondary Metabolism-Inducing Treatments During in vitro Development of Turmeric (Curcuma longa L.) Rhizomes. J Herbs Spices Med Plants 15: 303-317.

Daniel M, Sabnis SD (1978) Chemical Systematics of Family Gentianaceae. Curr Sci 47: 109-111.

Ellnain-Wojtaszek M, Zgorka G (1999) High-performance liquid chromatography and thin-layer chromatography of phenolic acids from Ginkgo biloba L. - leaves collected within vegetative period. J Liq Chromatogr R T 22: 1457-1471.

Everitt BS, Landau S, Leese M, Stahl D (2011) Cluster Analysis. John Wiley \& Sons, Ltd.

Fisher RA (1935) The Design of Experiments. Oliver and Boyd Press, Edinburgh.

Gamborg OL, Miller RA, Ojima K (1968) Nutrient requirements of suspension cultures of soybean root cells. Exp Cell Res 50: 151-158.

Giberti S, Bertea CM, Narayana R, Maffei ME, Forlani G (2012) Two phenylalanine ammonia lyase isoforms are involved in the elicitorinduced response of rice to the fungal pathogen Magnaporthe oryzae. J Plant Physiol 169: 249-254.

Grzegorczyk I, Wysokińska H (2009) The effect of methyl jasmonate on production of antioxidant compounds in shoot cultures of Salvia officinalis L. Herba Pol 55: 238-243.

Hegnauer R (1966) Chemotaxonomie der Pflanzen, vol. 17. Birkhauser, Basel.

Jaremicz Z, Łuczkiewicz M, Kokotkiewicz A, Królicka A, Sowiński P (2014) Production of tropane alkaloids in Hyoscyamus niger (black henbane) hairy roots grown in bubble-column and spray bioreactors. Biotechnol Lett, DOI 10.1007/s10529-013-1426-9.

Jeeshna MV, Paulsamy S (2011) Evaluation of certain flavonoids of medicinal importance in the wild and micropropagated plants of the endangered medicinal species, Exacum bicolor Roxb. J Appl Pharm Sci 01: 99-102.

Jolliffe IT (2010) Principal component analysis. Second edition. Springer-Verlag New York Inc., New York.

Keil M, Härtle B, Guillaume A, Psiorz M (2000) Production of amarogentin in root cultures of Swertia chirata. Planta Med 66: 452-457.

Kempthorne O, Hinkelmann K (2007) Design and analysis of experiments. Vol. 1. Introduction to experimental design. John Wiley \& Sons, Hoboken, NJ, USA.

Khadem S, Marles RJ (2010) Monocyclic phenolic acids; hydroxy- and polyhydroxybenzoic acids: occurrence and recent bioactivity studies. Molecules 15: 7985-8005.
Kikowska M, Budzianowski J, Krawczyk A, Thiem B (2012) Accumulation of rosmarinic, chlorogenic and caffeic acids in in vitro cultures of Eryngium planum L. Acta Physiol Plant 34: 2425-2433.

Krolicka A, Szpitter A, Gilgenast E, Romanik G, Kaminski A, Lojkowska E (2008) Stimulation of antibacterial naphthoquinones and flavonoids accumulation in carnivorous plants grown in vitro by addition of elicitors. Enzyme Microb Tech 42: 216-221.

Kruskal WH, Wallis WA (1952) Use of Ranks in One-Criterion Variance Analysis. J Am Stat Assoc 47: 583-621.

Kumar V, Chauhan RS, Sood H (2013) In vitro production and efficient quantification of major phytopharmaceuticals in an endangered medicinal herb, Swertia chirata. Int J Biotech Bioeng Res 4: 495-506.

Levene H (1960) Robust tests for equality of variances. In In Contributions to Probability and Statistics: Essays in Honor of Harold Hotelling. Olkin I, Ghurye SG, Hoeffding W, Madow WG and Mann HB, eds, pp 278-292. Stanford University Press, Stanford.

Mothana RAA, Grunert R, Lindequist U, Bednarski PJ (2007) Study of the anticancer potential of Yemeni plants used in folk medicine. Pharmazie 62: 305-307.

Mothana RAA, Lindequist U (2005) Antimicrobial activity of some medicinal plants of the island Soqotra. J Ethnopharmacol 96: 177-181.

Mothana RAA, Mentel R, Reiss C, Lindequist U (2006) Phytochemical screening and antiviral activity of some medicinal plants from the Island Soqotra. Phytotherapy Res 20: 298-302.

Murashige T, Skoog F (1962) A revised medium for rapid growth and bio assays with tobacco tissue cultures. Physiol Plant 15: 473-497.

Namdeo AG (2007) Plant cell elicitation for production of secondary metabolites. Pharmacogn Rev 1: 69-79.

Qu JG, Zhang W, Yu XJ (2011) A combination of elicitation and precursor feeding leads to increased anthocyanin synthesis in cell suspension cultures of Vitis vinifera. Plant Cell Tiss Org 107: 261-269.

Riedel H, Akumo DN, Saw NMMT, Kutuk O, Neubauer P, Smeta I (2012) Elicitation and precursor feeding influence phenolic acids composition in Vitis vinifera suspension culture. Afr J Biotechnol 11: 3000-3008.

Riseman A (2006) Exacum. In Flower Breeding and Genetics. Anderson NO, eds, pp 601-622. Springer Netherlands.

Shapiro SS, Wilk MB (1965) An analysis of variance test for normality (complete samples). Biometrika 52: 591-\&.

Skrzypczak-Pietraszek E, Pietraszek J (2012) Chemical profile and seasonal variation of phenolic acid content in bastard balm (Melittis melissophyllum L., Lamiaceae). J Pharmacent Biomed Anal 66: 154-161.

Skrzypczak-Pietraszek E, Szewczyk A, Piekoszewska A, Ekiert H (2005) Biotransformation of hydroquinone to arbutin in plant in vitro cultures preliminary results. Acta Physiol Plant 27: 79-87.

Thiem B, Krawczyk A (2010) Enhanced isoflavones accumulation in methyl jasmonate-treated in vitro cultures of kudzu (Puerara lobata Ohwi). Herba Pol 56: 48-56.

Weremczuk-Jeżyna I, Grzegorczyk-Karolak I, Frydrych B, Królicka A, Wysokińska H (2013) Hairy roots of Dracocephalum moldavica: rosmarinic acid content and antioxidant potential. Acta Physiol Plant 35: 2095-2103.

Yuan YM, Wohlhauser S, Moller M, Klackenberg J, Callmander MW, Kupfer P (2005) Phylogeny and biogeography of Exacum (Gentianaceae): A disjunctive distribution in the Indian Ocean Basin resulted from long distance dispersal and extensive radiation. Syst Biol 54: $21-34$. 